\title{
Migration Processes and Volatiles Delivery
}

\author{
Mikhail Marov \& Sergei Ipatov \\ M. V. Keldysh Institute, Miusskaya sq. 4, Moscow 125047, Russia
}

\begin{abstract}
Migration processes of comets and asteroids from the outer regions of the solar system, including the Edgeworth-Kuiper belt, are regarded as important mechanisms for the formation and evolution of the inner planets. These minor bodies may be responsible for the delivery of volatile matter to the inner planets and thus be responsible for the origin of life. We estimate that the cumulative mass of icy comets impacting on the Earth during the formation of the giant planets is similar to the mass of water in the Earth oceans, and that Mars acquired more water per unit planet mass than Earth. We find that these cometary objects mostly evolved from typical near-Earth orbits and Encke-type orbits with aphelia located inside the orbit of Jupiter, and played a greater role than those with Jupiter-crossing orbits. The relative importance of comets and chondrites in the delivery of volatiles is constrained by the observed fractionation patterns of atmospheric noble gas abundance.
\end{abstract}

\section{Introduction}

Comets and asteroid chondrites are remnants of the formation of the outer planets, and contain primordial material from which the planets were built, and comets which are tidally perturbed from the Oort cloud into the inner solar system may contain pristine material from the presolar cloud. When these bodies collide with planets, they can deliver volatiles and organic or prebiotic compounds to the planets, thereby depositing on the planets the fundamental building-blocks for life (Marov \& Ipatov 2001).

Unlike the giant planets which are preserved essentially unmodified from the pre-solar nebula, the inner planets contain heavier and cosmically less abundant elements in an iron-silicate matrix. Three mechanisms are thought to have operated concurrently to produce this: uneven fractionation and condensation in the accretionary disk; unequal degree of degassing of the composed matter; and heterogeneous accretion (Marov \& Grinspoon 1988). Uneven condensation should result in a lack of low temperature volatiles (nitrogen, water, sulfur, carbon) in the region of terrestrial planets with formation at temperatures of up to $1500 \mathrm{~K}$ and pressures less than $10^{-1}$ mbar.

After the formation of the bulk of planetary mass, during the final accretionary phase, asteroid-size bodies consisting of the last low-temp condensates (similar to most primitive chondritic meteorites, enriched in hydrated silicates and trapped gases) are believed to have fallen onto the inner planets. The relative contribution of either endogenous (outgassing) or exogenous (asteroid collisions) sources is difficult to assess, although it is constrained by the pattern of noble gas abundances in the planetary atmospheres (Owen \& Bar-Nun 2001). 


\section{A model for the migration of Jupiter-crossing objects}

We have developed a model to simulate the dynamics of small bodies that migrate inwards to cross the orbits of the inner planets. Our model starts by considering the migration of planetesimals in the feeding zones of the giant planets and trans-Neptunian objects (TNOs) to become Jupiter-crossing objects (JCOs), and then considers the orbital evolution of JCOs.

Evolution of 13000 virtual JCOs under the gravitational influence of all the planets, except for Pluto (and in some cases Mercury) were numerically modelled for intervals $T_{S} \geq 10 \mathrm{Myr}$. After $10 \mathrm{Myr}$, testing was carried out to determine whether some of the remaining objects could still evolve into orbits inside Jupiter's orbit. If so, the calculations were continued.

We performed several series of runs. In the first series $(n 1), 3100$ bodies having orbits close to those of 20 real Jupiter-family comets with periods $5<P_{a}<9$ yr were modelled. In other series, initial orbits were chosen close to those of several known comets $(2 \mathrm{P}, 9 \mathrm{P}, 10 \mathrm{P}, 22 \mathrm{P}, 28 \mathrm{P}$ or $39 \mathrm{P})$. The probabilities of collision of these objects with the inner planets, and times spent in different orbits (e.g., Aten and Apollo orbits), during the lifetime of an object were calculated based on their orbital elements calculated with time steps of $500 \mathrm{yr}$. From these calculations, we deduced the mean probabilities and respective collision times for all the objects.

To achieve this, we used the Bulirsh-Stoer method (BULSTO code) with the integration step error less than $\varepsilon \in\left[10^{-9}-10^{-8}\right]$ and in some runs $\varepsilon \leq 10^{-12}$, or a symplectic method (RMVS3 code) from the integration package of Levison \& Duncan (1994). Both the symplectic method at an integration step $d_{s} \leq 10$ days and BULSTO code essentially gave similar values for the mean collision probabilities and characteristic times spent in different orbits for the above mentioned $\varepsilon$ values and with similar initial data. The two methods gave different values for the probability of collisions with the Sun but gave the same value for collisions with the planets. Details of the procedure and runs performed are described by Ipatov \& Mather (2003).

The results for evolution of the objects showed considerable variation between different runs. A few $(\sim 0.1 \%)$ bodies, mainly in the series with $2 \mathrm{P}$ and $10 \mathrm{P}$ orbits, achieved and retained typical Apollo orbits with a semi-major axis $a<2 \mathrm{AU}$, or even Amor or inner-Earth object (IEO) orbits for $10^{6}-10^{8}$ years. Figure 1 shows a pattern of decoupling of a body from Jupiter for several Myr. Several objects were shown to move inside Jupiter's orbit for much longer. For the $n 1$ series, the mean collision probability with the Earth was found to be $P_{E}>4.5 \times 10^{-6}$. The mean value for all series with RMVS3 code is about an order of magnitude higher. In one JCO series, $P_{E}$ varied from $10^{-6}$ (39P with BULSTO) to more than $10^{-4}$ (2P with both BULSTO and RMVS3).

Collision probabilities with the inner planets, and time residences in Apollo, Aten and IEO orbits, were similar for all runs, if those reaching perihelion distances $q$ less than several radii $r_{S}$ of the Sun were omitted. If orbits for the comets $2 \mathrm{P}$ and $10 \mathrm{P}$ with $q<2 r_{S}$ were included, resulting in some collisions with the Sun, objects with inclination $i>90^{\circ}$ were produced. No such objects resulted if $q<2 r_{S}$ orbits were omitted. The specific mass of matter delivered by JCOs to an inner planet (normalized to its mass) turned out nearly the same for Earth and Venus though greater for Mars. 

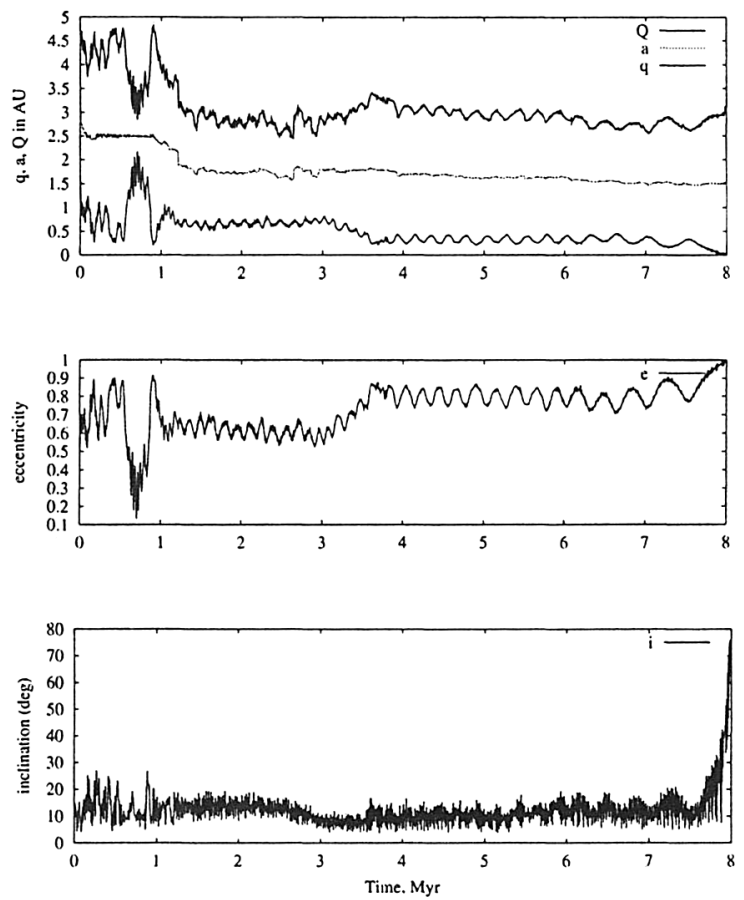

Figure 1. Time variations in $q=a(1-e), a, Q=a(1+e), e$, and $i$ for a former JCO in initial orbit close to that of Comet 10P

\section{Volatiles Inventory}

Based on the estimated mean collision probability of $P_{E}=4 \times 10^{-6}$ and assuming that the total mass of planetesimals $M_{p l}$ that crossed Jupiter's orbit is $\sim 100 m_{\oplus}$ ( $m_{\oplus}=$ mass of the Earth), we find that the total mass of bodies which impacted the Earth is $4 \times 10^{-4} m_{\oplus}$. If ices represent half of this mass, then the total mass of ice that was delivered to the Earth from the feeding zone of the giant planets is roughly equal to the mass of the Earths oceans. During the following $4 \mathrm{Gyr}$ the effectiveness of transport was much lower and less than $0.1 \%$ of the total mass of volatiles was delivered. The specific mass of the volatiles accumulated by a planet by comets from JCOs is greater for Mars than for Earth and Venus which supports the idea of a relatively large ancient ocean on this planet.

Our estimate of the water inventory to the early Earth is greater than those of Morbidelli et al. (2000) and Levison et al. (2001). This discrepancy is probably because (a) they used a relatively small number of objects in their modelling, (b) they did not consider migration of bodies into the orbits with aphelion distance $Q<4.2 \mathrm{AU}$ and $q<1 \mathrm{AU}$, and (c) Levison et al. (2001) did not take into account the influence of the terrestrial planets. Morbidelli et al. (2000) obtained $P=(1-3) \times 10^{-6}$ in the 5-8 AU region, which is close to some of our estimates. Their value of $M_{p l}$ is smaller, possibly because they neglected migration of planetesimals caused by the gravitational influence of Jupiter. 
The fractionation pattern of noble gas abundance measured in the atmospheres of Earth, Mars, and Venus is regarded as an important constraint on the relative importance of comet and asteroid chondrites in the delivery of volatiles. Laboratory experiments on the trapping of gases in amorphous ice forming at low temperature (Owen \& Bar-Nun 2001) suggest that this is an important mechanism for delivering noble gases to planets. The relative abundance pattern of argon, krypton, and xenon in the atmospheres of inner planets may then be partially explained if the efficiency of gas trapping is strongly dependent on temperature. Similarly, the D/H ratio of $\mathrm{H}_{2} \mathrm{O}$ molecules in Comets Halley, Hyakutake, and Hale-Bopp is nearly twice that of seawater. This discrepancy may be caused by the deuterium enrichment near Jupiter being different from that in more distant colder regions in the period before the heavy bombardment of the inner planets took place (Delsemme 1999). While the relative importance of internal degassing compared to the input of volatile-rich materials from outer regions of the solar system remains unclear, heterogeneous accretion is thought to be an important factor in the observed abundance of volatiles in the current atmospheres of the planets.

\section{Conclusion}

We find that both endogenous and exogenous processes are responsible for the volatile gases in the terrestrial planets. Our simulations show that collisions of planets with small objects are an important source of these volatiles, and that even a relatively small portion $(\sim 0.001)$ of JCOs which transit to orbits with aphelia inside Jupiter's orbit $(Q<4.2 \mathrm{AU})$ and occupy such orbits for more than $1 \mathrm{Myr}$, contribute significantly to the supply of material. The mean probability for a JCO to collide with the Earth is estimated to be as high as $P=4 \times 10^{-6}$. This probability implies that the total mass of volatiles delivered to the Earth from the feeding zone of the giant planets is similar to the mass of the Earth's oceans. Further advances in the fields of stochastic dynamics, migration and collisions of small bodies will allow us to better understand the problem of solar system origin and evolution and highlight the key problems in bioastronomy.

Acknowledgments. This work was supported by INTAS (00-240), NRC (0158730), NASA (NAG5-10776), and RFBR (01-02-17540).

\section{References}

Delsemme, A. H., 1999, Planet. Space Sci., 47, 125

Ipatov, S. I., \& Mather, J. M. 2003, Advances in Space Research, in press

Levison H. F., \& Duncan, M. J. 1994, Icarus, 108, 18

Levison, H. F., et al., 2001, Icarus, 151, 286

Marov, M. Ya., \& Grinspoon, D. 1998, The Planet Venus, Yale University Press

Marov, M. Ya., \& Ipatov, S. I., 2001, in Collisional Processes in the Solar System, ed. M. Ya. Marov \& H. Rickman (ASSL) 261, 223

Morbidelli, A., et al., 2000, Meteoritics \& Planetary Science, 35, 1309

Owen, T. C., \& Bar-Nun, A. 2001, in Collisional Processes in the Solar System, ed. M. Ya. Marov \& H. Rickman, (ASSL) 261, 249 